\title{
In Vivo and In Vitro Evidences of Dehydroepiandrosterone Protective Role on the Cardiovascular System
}

\author{
Tiphaine Mannic $^{1,2,} ;$ Joanna Viguie ${ }^{3}$; Michel Florian Rossier ${ }^{1,4}$ \\ ${ }_{1}^{1}$ Department of Human Protein Science, Faculty of Medicine, University of Geneva, Geneva, Switzerland \\ 2 Department of Genetics and laboratory Medicine, University Hospitals of Geneva, University of Geneva, Geneva, Switzerland \\ 3 Department of Neurosciences, Faculty of Medicine, University of Geneva, Geneva, Switzerland \\ ${ }^{4}$ Department of Neurosciences, Faculty of Medicine, University of Geneva, Geneva, Switzerland
${ }_{\text {Service of Clinical Chemistry and Toxicology, Central Institute of the Hospital of Valais, Sion, Switzerland }}$ \\ *Corresponding author: Tiphaine Mannic, Department of Genetics and laboratory Medicine, University Hospitals of Geneva, University of Geneva, Geneva, Switzerland. Tel: +41- \\ 223795775, Fax: +41-223795502, E-mail: Tiphaine.mannic@unige.ch
}

Received: October 17, 2014; Revised: December 5, 2014; Accepted: February 14, 2015

\begin{abstract}
Context: Dehydroepiandrosterone (DHEA) and its sulfate ester, Dehydroepiandrosterone Sulfate (DHEA-S) have been considered as putative anti-aging hormones for many years. Indeed, while DHEAS is the most abundant circulating hormone, its concentration is markedly decreased upon aging and early epidemiologic trials have revealed a strong inverse correlation between the hormone concentrations and the occurrence of several dysfunctions frequently encountered in the elderly. Naturally, hormonal supplementation has been rapidly suggested to prevent DHEA (S) deficiency and therefore, age-related development of these pathologies, using the same strategy as estrogen replacement therapy proposed in postmenopausal women.

Evidence Acquisition: All references were searched using PubMed and the following strategy: our initial selection included all articles in English and we sorted them with the following keywords: "DHEA or DHEA-S" and "heart or vascular or endothelium or cardiovascular disease". The search was limited to neither the publication date nor specific journals. The final selection was made according to the relevance of the article content with the aims of the review. According to these criteria, fewer than $10 \%$ of the articles retrieved at the first step were discarded.

Results: In this short review, we have focused on the cardiovascular action of DHEA. We started by analyzing evidences in favor of a strong inverse association between DHEA $(S)$ levels and the cardiovascular risk as demonstrated in multiple observational epidemiologic studies for several decades. Then we discussed the different trials aimed at supplementing DHEA (S), both in animals and human, for preventing cardiovascular diseases and we analyzed the possible reasons for the discrepancy observed among the results of some studies. Finally, we presented putative molecular mechanisms of action for DHEA(S), demonstrated in vitro in different models of vascular and cardiac cells, highlighting the complexity of the involved signaling pathways.

Conclusions: The identification of the beneficial cardiovascular effects of DHEA $(S)$ and a better understanding of the involved mechanisms should be helpful to develop new strategies or pharmacologic approaches for many lethal diseases in Western countries.
\end{abstract}

Keywords: Dehydroepiandrosterone; Endothelium; Myocytes, Cardiac; Cardiovascular System; Disease; Steroids

\section{Context}

An excess of steroid hormones such as mineralocorticoids has been linked to heart hypertrophy, arrhythmias, inflammation, fibrosis, and apoptosis as well as to endothelial and smooth muscle vascular dysfunctions. Benefits of antagonizing aldosterone action in patients with cardiovascular diseases (CVD) have demonstrated a causal relationship between steroid hormones and CVD (1). On the other hand, putatively protective steroid hormones such as dehydroepiandrosterone(DHEA) decrease with aging. DHEA, and particularly its sulfated derivative, DHEA sulfate (DHEA-S), are among the most abundant circulating steroid hormones. These two hormones are derived from cholesterol and are principally produced by the adrenal cortex; however, small amounts of them have been proposed to be produced by other organs such as the heart. Since the discovery of DHEA and its sulfated metabolite during the first half of the 20th century, many publications have hypothesized that the development of DHEA deficiencies with age might play a key role in the degradation of many functions and contribute to the genesis of several disorders, including cardiovascular defects (2). Other studies have reported a beneficial role for DHEA (S) in various physiologic or pathophysiologic conditions such as brain development, aging, osteoporosis, immune system-mediated rheumatologic diseases, diabetes mellitus (DM), obesity, chronic heart failure (particularly when linked with oxidative stress), and recently, vascular remodeling as occurring in pulmonary arterial hypertension (3). Nevertheless, only few marked beneficial effects have been clearly reported with DHEA

Copyright (C) 2015, Research Institute For Endocrine Sciences and Iran Endocrine Society. This is an open-access article distributed under the terms of the Creative Commons Attribution-NonCommercial 4.0 International License (http://creativecommons.org/licenses/by-nc/4.0/) which permits copy and redistribute the material just in noncommercial usages, provided the original work is properly cited. 
supplementation in human and therefore, this subject is still controversial.

In the present article, we aimed at providing a short review of recently published works showing the inverse correlation between the circulating levels of DHEA and DHEA-S and the degree of illness in several pathophysiologic situations. We focused our attention on CVD and on the beneficial effects of counteracting DHEA (S) deficiency by supplementation. We also discussed the putative mechanisms of action exerted by the hormone in several cardiovascular target organs and tissues.

\section{Evidence Acquisition}

All references have been searched using PubMed and the following strategy:

Our initial selection included all articles in English language and we sorted them with the keywords "DHEA or DHEA-S" and "heart or vascular or endothelium or cardiovascular disease". The search was not limited by neither the publication date nor specific journals. The final selection was made according to the relevance of the article content to the aims of the review. According to these criteria, $<10 \%$ of the retrieved articles at the first step was discarded.

\section{Results}

\subsection{Low Levels of Dehydroepiandrosterone (Sul-} fate) Predicts Cardiovascular Health Disorders

\subsubsection{Assessing a Role for Dehydroepiandrosterone (Sul- fate)}

Correlation between DHEA or DHEA-S plasma levels and ageing-related pathologies has been widely explored during the last twenty years. Indeed, DHEA (S) levels in circulation decrease with ageing more markedly than those of other hormones. Numerous studies have shown a negative correlation between the levels of these hormones and ageing, as defined by the occurrence of several disorders including CVD as well as more definitive entities such as mortality. However, the predictive value is influenced by factors such as sex and smoking habits. In a cohort of elderly subjects, it was demonstrated that DHEA-S levels inversely correlated with men mortality whereas there was no association with women mortality (4). In addition, only one published work has clearly mentioned physiologic differences between DHEA and DHEA-S to relate their respective concentrations to various pathologies (5). They highlighted the difficulty to properly correlate DHEA concentration with diseases, probably due to the different hormone distribution throughout life, compared to its sulfated form. The authors also reported that DHEA$S$ levels are much more often measured in studies than DHEA levels, which are often not reported at all.

\subsubsection{Dehydroepiandrosterone (Sulfate) Deficiency and} Cardiovascular Risk

The CVD encompass numerous and diverse kinds of pathologies related to peripheral vessels as well as to the heart. Barrett-Connor et al. have demonstrated that mean DHEA-S concentrations decreases in men with age and are significantly lower in those with a history of heart disease (6). After adjustment for age and other CVD risk factors, they found that men with lower DHEA-S levels (< $3.8 \mu \mathrm{mol} / \mathrm{L}$ ) had a 1.5-fold higher risk to die of any cause (which was not statistically significant), but a 3.3-fold higher risk to die of CVD $(\mathrm{P}<0.05)$, and particularly from ischemic heart disease (IHD).

Clinical and demographic parameters are relevant to drawing any conclusion about causal relationship between DHEA (S) and CVD. For example, concerning the sex, studies are more consistent for men, and when looking at women cohorts, the relationship between DHEA-S and mortality from all causes, including from CVD, is often less obvious. Nevertheless, in the WISE (Women's Ischemia Syndrome Evaluation) study, including 270 postmenopausal women undergoing coronary angiography for suspected ischemia, a positive correlation between DHEA-S levels and CVD outcome was reported, which was potentially explained by a better atherosclerosis state of patients with higher DHEA-S levels (7). Another study demonstrated that the decrease of DHEA-S concentrations observed in elderly women is significantly more pronounced in case of coronary heart diseases, osteoporosis or depression (8).

In contrast, prospectively investigating the longitudinal patterns of sex steroids in a cohort of 254 elderly men on a ten-year follow-up did not reveal any significant association between sex steroids (testosterone and DHEA-S) and the incidence of CVD or all-cause mortality risk (9). In conclusion, DHEA-S levels are variably good markers not perfect for predicting CVD in the general population probably because many additional important factors have to be integrated in these predictions.

The role of DHEA and DHEA-S has been studied in the context of endothelial function in postmenopausal women with coronary risks factors. Results have shown that lower levels of DHEA-S are associated with endothelial dysfunction as assessed by a positive correlation between DHEA-S and percentage of flow-mediated dilatation (\% FMD), a marker of endothelial function (10). This correlation was independent of other coronary risk factors such as age, body mass index, blood pressure, total and highdensity lipoprotein cholesterol (HDL-C), fasting glucose levels, or smoking habits.

Recent studies evaluated whether DHEA $(S)$ is associated with development of carotid atherosclerosis, a situation predicting cardiovascular complications. For example, measurement of intima media thickness (IMT), blood flow volume, and endothelial function (\%FMD) has been performed by ultrasonography of carotid arteries in 419 
Japanese patients of both sexes with cardiovascular risk factors, and IMT appeared to be inversely correlated with DHEA-S levels in males, but not in females. In contrast, DHEA-S was a positive factor for predicting blood flow volume in females, but not in males, and no significant correlation could be demonstrated in this study between DHEA-S and \%FMD regardless of sex (11).

The association between androgen levels, including DHEA-S and total and bioavailable testosterone, with aortic atherosclerosis has been also investigated in 1032 nonsmoking Dutch men and women aged 55 years or older. Intimal atherosclerosis was assessed by radiographic detection of calcified deposits in the abdominal aorta; however, no clear association between levels of DHEA-S and presence of severe aortic atherosclerosis was found in men or women (12).

In order to examine the association between DHEA and coronary atherosclerosis, plasma DHEA and DHEA-S levels had been previously determined in 206 middle-aged patients undergoing coronary angiography. The DHEA$S$ level was significantly lower in subjects with stenosis and was inversely associated with the number of affected coronary vessels and the extent of coronary atherosclerosis (13). Another part of the same study also investigated the putative protection of DHEA against the development of vasculopathy after cardiac allograft. In a cohort of 61 cardiac allograft recipients, it has been found that DHEA levels inversely correlated with the development of accelerated coronary vasculopathy, which appeared earlier in patients with low levels of the hormone, suggesting that high levels of DHEA could retard the development of coronary atherosclerosis and coronary allograft vasculopathy.

In a prospective study (Massachusetts Male Aging Study) on 1167 males aged 40 to 70 years at baseline followed for over ten years, low serum DHEA(S) was predictive of later IHD independent of other risk factors. The subjects in the lowest quartile (DHEA-S $<4.3 \mu \mathrm{mol} / \mathrm{L}$ ) were significantly more likely to develop IHD with an adjusted odds ratio of 1.6 (95\% CI, 1.07-2.39) (14).

The risk of ischemic stroke might be influenced by DHEA$S$ levels, as suggested by another study based on a cohort of 32826 women (Nurses' Health Study) following patients for cardiovascular events (15). Matching 461 cases of ischemic strokes confirmed by medical records, with controls of the same age, race, menopausal status, hormone use, and smoking status demonstrated that lower DHEA-S levels were associated with a greater risk of stroke.

Association of atrial fibrillation and its prevalence with DHEA-S levels were investigated in 436 men and 544 women aging 65 to 97 years old. Interestingly, a low DHEA-S concentration was significantly associated with cardiac arrhythmias in men, while occlusive arterial diseases, chronic obstructive lung disease, or osteoporosis were more frequent in women with low DHEA-S. However, only the association with osteoporosis remained significant after controlling for differences in lifestyle and general health status parameters (16). The authors concluded that in the elderly, low serum DHEA-S levels are more a nonspecific indicator of aging and health status rather than a risk marker of specific diseases.

Diabetes mellitus is frequently associated with CVD, and significantly lower DHEA-S concentrations were observed in 51 diabetic patients with a specific subtype of type $2 \mathrm{DM}$ in comparison with 49 healthy controls (17). The putative cardioprotective effect of DHEA has been investigated in 62 male patients with type 2 DM by determining the association between the hormone levels and the activated protein C, C-reactive protein, and the IMT of the carotid artery. The study concluded that lower circulating levels of DHEA are associated with decreased activated protein $C$ generation and higher IMT in patients type $2 \mathrm{DM}(18)$.

Finally, testosterone and DHEA were determined in 153 men with DM (age, $65 \pm 9$ years) with coronary artery disease (CAD). Androgen deficiency, defined as serum levels below the 10th percentile of healthy peers, was common in men with DM with stable CAD and predicted increased cardiovascular mortality (19).

\subsection{In Vivo Dehydroepiandrosterone Supplemen- tation: Is it Efficient to Prevent Cardiovascular Dis- eases?}

Several early supplementation studies have been done before any clinical studies aimed at measuring DHEA or DHEA-S levels. This fact led to many misunderstandings as supplementation was not always provided for correcting DHEA(S) deficiencies in patients.

\subsubsection{Supplementation in Animal Models}

Systemic vascular remodeling, as occurring during restenosis after injury, is characterized by the presence of proliferative and apoptosis-resistant smooth muscle cells with activated Akt pathway. Because DHEA has been previously proposed to inhibit this pathway in cancer, Bonnet et al. investigated a putative role for DHEA in remodeling vessel wall both in vitro, using cultured human carotid vascular smooth muscle cells and saphenous vein grafts challenged with PDGF, and in vivo, with a rat carotid injury model. In each case, DHEA reduced proliferation and increased apoptosis of smooth muscle cells through the inhibition of the Akt pathway. DHEA effect was unaffected by androgen or estrogen antagonists, suggesting a steroid receptor-independent mechanism. The authors concluded that orally available DHEA might be attractive for treating systemic vascular remodeling, including restenosis (20).

In order to mimic the metabolic dysregulation often occurring in the elderly, animals are often submitted to a high fat diet. These animals develop inflammatory processes and cardiomyopathies linked to their diabetic state. In this context, DHEA seems to be beneficial for counteracting cardiac disturbances such as unbalanced oxidative status, impairment of cardiac myogenic 
factors, and contractility dysfunction (21). In a model of rabbits fed with a high fat diet and displaying hyperglycemia, insulin resistance, dyslipidemia, increased oxidative stress, and proinflammatory insults, the impairment of cardiac contractility was observed by measuring the switch of myosin heavy chain (MHC) isoforms expression. In these animals, low dose of DHEA supplementation, which did not affect glucose levels or insulin resistance, restored the oxidative balance and lowered lipid levels and inflammation, preventing alteration of the cardiac function and delaying the onset of cardiac tissue damage. A similar effect of DHEA has been observed by the same authors in rats with streptozocin-induced DM (22). The hyperglycemia-induced oxidative stress increases advanced glycated end-products (AGEs) and triggers a cascade of signaling involving NFKB (23), finally leading to interstitial fibrosis. The DHEA treatment, by improving the oxidative balance, counteracts the AGE action and restores the contractility of the ventricular tissue by preventing the fibrotic response.

DHEA also improved the right ventricular function and remodeling in rats upon reoxygenation after 21 days of hypoxia (24). Ex vivo investigations demonstrated that the presence of DHEA during the reoxygenation phase increased myocytes proliferation through Cyclic AMP Response Element Binding Protein (CREB) and PGC1 $\alpha$ activation, prevented mitochondrial fragmentation, and improved the respiratory chain activity. It also reduced the cellular production of superoxide anions.

\subsubsection{Supplementation in Clinical and Epidemiological Studies}

DHEA supplementation ( $25 \mathrm{mg} /$ day) in asymptomatic, normotensive, non-diabetic and nonsmoker men with hypercholesterolemia (mean age, 54 years) increased plasma levels of DHEA-S and significantly ameliorated the \%FMD of arteries (25). This observation points out that for this specific DHEA (S) action, results are in good agreement in both sexes, as previously discussed for postmenopausal women (10). This was confirmed in another study, where supplementation of 36 healthy postmenopausal women with DHEA (100 mg/day) for three months also increased the \%FMD and the laser Doppler velocimetry parameters, and significantly reduced total plasma cholesterol concentration (26).

In contrast, DHEA-S supplementation (50 mg/day) appeared to have beneficial effect on neither insulin action, determined by euglycemic hyperinsulinemic clamp, nor the quality of life, measured by extensive parameters in female patients with hypopituitary hypoadrenalism (with very low DHEA-S levels) and following standard replacement therapy (27). Only triglycerides concentrations were significantly lower following DHEA-S treatment. However, the quality of life in this study was not assessed in term of cardiovascular health, which has been clearly shown to be associated with metabolic diseases.
The benefits of DHEA supplementation in the aging population is highly controversial within the literature and therefore, published studies have been recently reviewed in a meta-analysis to determine whether there is a potential therapeutic role of DHEA in postmenopausal women (28). In spite of several observational studies showing that lower DHEA levels were associated with increased cardiovascular risk in women, interventional trials did not demonstrate improvement atherosclerosis or cardiovascular risk factors by DHEA supplementation. The DHEA even appears to be responsible for lowering HDL-C levels, a putative cardioprotective marker, probably through an androgen-dependent mechanism; however, it is not clear how much this action of DHEA is detrimental to the cardiovascular function. The authors conclude that there was no strong current evidence in favor of DHEA supplementation in healthy postmenopausal women.

Several reports also suggest that high doses of DHEA (> $25 \mathrm{mg} /$ day) could be potentially pro-arrhythmogenic (29) and in most cases, palpitations or tachycardia disappear when the drug is stopped. Whether this deleterious effect of DHEA is related to its in vivo transformation into androgens, or to a direct action of the hormone on cardiomyocytes, remains to be determined.

\subsection{In Vitro Investigation of Cellular and Molecu- lar Mechanisms of Dehydroepiandrosterone (Sul- fate) Cardiovascular Action}

When assessing the cellular and molecular mechanism of DHEA (S) action, it is important to realize that because of their quite different chemical properties, DHEA and DHEA-S may act differently on their targets cells in terms of both efficiency and specificity of the involved signaling pathways. Moreover, both genomic and non-genomic mechanisms can be recruited by these hormones with divergent consequences for the cell function.

\subsubsection{Dehydroepiandrosterone Effect on Isolated Endo- thelial Cells}

Several effects of DHEA (and not always mimicked by DHEA-S) on the endothelium have been extensively described (Table 1). The formation of the atheroma plaque, for example, requires the adhesion of monocytes to vascular endothelium through binding of endothelial neural cell adhesion molecule (NCAM) to monocyte NCAM. This interaction is prevented by sialylation of NCAM. When administered to cultured human coronary artery endothelial cells, DHEA inhibited monocyte adhesion by increasing the expression of polysialylated NCAM, a response sensitive to androgen receptor (AR) and estrogen receptor (ER) antagonists in a sex-dependent manner (30). Interestingly, the expression of polysialylated NCAM was completely abolished in the presence of trilostane, an inhibitor of $3 \beta$-hydroxysteroid dehydrogenase, sug gesting that DHEA acts via its end metabolites, estradiol and testosterone. 
Table 1. In Vitro or Ex Vivo Assessment of Dehydroepiandrosterone (Sulfate) Mode of Action on Various Cardiovascular Target Tissues ${ }^{a}$

\begin{tabular}{lcccc}
\hline $\begin{array}{l}\text { Target Tissue/ } \\
\text { Cell (Species) }\end{array}$ & $\begin{array}{c}\text { Macroscopic Biologic Re- } \\
\text { sponse to DHEA (S) }\end{array}$ & $\begin{array}{c}\text { Receptor Involved } \\
\text { (or Messenger) }\end{array}$ & $\begin{array}{c}\text { Cellular Signaling Pathway or Molecular } \\
\text { Mechanism Suggested }\end{array}$ & References \\
\hline Endothelial & & &
\end{tabular}

Endothelial

cells

\begin{tabular}{|c|c|c|c|c|}
\hline $\begin{array}{l}\text { Coronary ar- } \\
\text { tery (human) }\end{array}$ & $\begin{array}{l}\text { Inhibition of monocyte adhe- } \\
\text { sion }\end{array}$ & $\begin{array}{l}\text { AR and ER (via testos- } \\
\text { terone or estradiol) }\end{array}$ & Increased expression of polysialylated NCAM & (30) \\
\hline $\begin{array}{l}\text { Umbilical } \\
\text { vein/HUVEC } \\
\text { (human) }\end{array}$ & $\begin{array}{l}\text { Inhibition of monocyte adhe- } \\
\text { sion and of TNF } \alpha \text {-induced } \\
\text { inflammation }\end{array}$ & $\mathrm{N} / \mathrm{D}$ & $\begin{array}{l}\text { Inhibition of NFKB translocation, reduction of } \\
\text { ICAM-1 and E-selectin expression, diminution of } \\
\text { ROS production }\end{array}$ & (31) \\
\hline $\begin{array}{l}\text { Umbilical } \\
\text { vein/HUVEC } \\
\text { (human) }\end{array}$ & $\begin{array}{l}\text { Prevention of monocyte adhe- } \\
\text { sion induced by high glucose }\end{array}$ & $\mathrm{N} / \mathrm{D}$ & $\begin{array}{l}\text { Inhibition of NFKB translocation and adhesion } \\
\text { molecule expression, antioxidant properties of } \\
\text { DHEA }(S)\end{array}$ & (32) \\
\hline Aorta (human) & $\begin{array}{l}\text { Inhibition of TNF } \alpha \text {-induced } \\
\text { inflammation }\end{array}$ & $\operatorname{PPAR} \alpha$ & $\begin{array}{l}\text { Attenuation of IL-8, ICAM-1 and VCAM-1 expres- } \\
\text { sion, inhibition of NFאB translocation }\end{array}$ & (33) \\
\hline $\begin{array}{l}\text { Umbilical } \\
\text { vein/HUVEC } \\
\text { (human) }\end{array}$ & Inhibition of cell proliferation & $\begin{array}{l}\text { NOT through AR or } \\
\text { ER (not DHEA-S) }\end{array}$ & $\mathrm{N} / \mathrm{D}$ & $(34)$ \\
\hline $\begin{array}{l}\text { Aorta (bovine) } \\
\text { and HUVEC } \\
\text { (human) }\end{array}$ & $\begin{array}{l}\text { Increase of cell proliferation } \\
\text { and protection against super- } \\
\text { oxide injury }\end{array}$ & $\begin{array}{l}\text { NOT through AR } \\
\text { or ER }\end{array}$ & $\begin{array}{l}\text { Antioxidant properties of DHEA }(S) \text {, increase of } \\
\text { ERK 1,2 and eNOS expression }\end{array}$ & $(26,35)$ \\
\hline $\begin{array}{l}\text { Aorta (bovine) } \\
\text { and HUVEC } \\
\text { (human) }\end{array}$ & Increase of NO release & $\begin{array}{l}\text { NOT through ER cell } \\
\text { surface GPCR }\end{array}$ & $\begin{array}{l}\text { NOS activation through acute (non-genomic) } \\
\text { and Pertussis toxin-sensitive pathway }\end{array}$ & $(3,36)$ \\
\hline Aorta (rats) & $\begin{array}{l}\text { Preservation of endothelial } \\
\text { function upon aortic stenosis }\end{array}$ & Sigma-1R & $\begin{array}{l}\text { Akt and eNOS phosphorylation and expression, } \\
\text { inhibition of Sig-1R downregulation }\end{array}$ & (37) \\
\hline
\end{tabular}

\section{Vascular \\ smooth \\ muscle cells}

Pulmonary

Relaxation of precontracted

and aortic

artery (rats)

\begin{tabular}{|c|c|c|c|}
\hline $\begin{array}{l}\text { Pulmonary } \\
\text { artery (rats) }\end{array}$ & $\begin{array}{l}\text { Reduction of hypotoxic } \\
\text { pulmonary hypertension, } \\
\text { relaxation of artery rings }\end{array}$ & $\mathrm{N} / \mathrm{D}$ & $\begin{array}{l}\text { Upregulation of the soluble guanylyl cyclase } \\
\text { (increased sensitivity to NO) }\end{array}$ \\
\hline
\end{tabular}

Pulmonary

artery (rats)

Relaxation of precontracted

NOT through A

Pulmonary ar- Inhibition of cell proliferation tery (human) and sensitization to apoptosis or ER

N/D

Inhibition of T-type calcium channels through a Pertussis toxin-sensitive $G$ protein

Inhibition of STAT3 and NFAT, induction of mitochondria depolarization and reduction of cell calcium concentration

\section{Cardiomyo-}

cytes

(PAH patients)

\begin{tabular}{|c|c|c|c|c|}
\hline Ventricle (rat) & $\begin{array}{l}\text { Reduction of hypertrophy } \\
\text { induced by endothelin }\end{array}$ & $\mathrm{N} / \mathrm{D}$ & Inhibition of BNP expression & $(42)$ \\
\hline Ventricle (rats) & $\begin{array}{l}\text { Prevention of corticosteroid- } \\
\text { induced hypertrophy and } \\
\text { chronotropic response }\end{array}$ & $\mathrm{N} / \mathrm{D}$ & $\begin{array}{c}\text { Decrease of T-type calcium channel expression } \\
\text { and activity, inhibition of ANP and BNP expres- } \\
\text { sion; antioxidant properties of DHEA }\end{array}$ & $(43)$ \\
\hline Ventricle (rats) & $\begin{array}{l}\text { Improvement of cardiac func- } \\
\text { tion and remodeling after } \\
\text { chronic hypoxia }\end{array}$ & $\mathrm{N} / \mathrm{D}$ & $\begin{array}{l}\text { Activation of CREB and PGC1 } \alpha \text {, increase of myo- } \\
\text { cyte proliferation and respiratory chain activity, } \\
\text { reduction of superoxide anions }\end{array}$ & $(24)$ \\
\hline
\end{tabular}


The protective action of DHEA against atherosclerosis has been further investigated in human umbilical vein endothelial cells (HUVEC), where the hormone $(100 \mu \mathrm{mol} / \mathrm{L})$ appeared to inhibit in vitro TNF $\alpha$-induced inflammatory response including $\mathrm{NF} \kappa \mathrm{B}$ translocation, ICAM-1 and E-selectin expression, reactive oxygen species (ROS) production, and U937 monocyte adhesion (31). The same group previously showed that DHEA was able to inhibit HUVEC proliferation by arresting the cell cycle in the G1 phase, a process that is over-activated in pathologic inflammatory and angiogenic conditions (34). It is noteworthy that the antiproliferative action of DHEA was not inhibited by AR or ER antagonists, was not mimicked by estradiol or testosterone, and more surprisingly, was not mimicked either by the sulfated ester, DHEA-S.

This effect of DHEA on the reduction of endothelial proliferation is in contrast with the observation by others that like estradiol and testosterone, DHEA increased the proliferation of bovine aortic endothelial cells and HUVEC (26). In this case, while the proliferative effect of estradiol and testosterone were abolished by the ER antagonist (ICI 182780) and AR antagonist (flutamide) none of them blocked the proliferative action of DHEA.

The reasons for the existing discrepancy between this finding and the previous study in term of endothelial cell proliferation are not known. High complexity of this system was suggested by an independent observation that testosterone induced the growth of human vascular endothelial cells via AR-independent mechanisms, while dihydrotestosterone (DHT) inhibited the growth of the same cells through an AR-dependent mechanism (35). Moreover, both testosterone and DHT enhanced vascular smooth muscle cell proliferation in a same way and ERK1/2 activation via AR-dependent and AR-independent pathways, respectively.

A protective action of DHEA on human vascular endothelial cells against superoxide injury has been also reported, and this effect appeared to be through AR-independent mechanisms (35), reminding the antioxidant properties of the DHEA molecule. Among other beneficial effects attributed to DHEA, its ability to stimulate nitric oxide (NO) release from endothelial cells is noteworthy. Indeed, DHEA stimulates NO release within five minutes exposure of intact bovine aortic endothelial or HUVEC (36). Increased NO release is due to activation of eNOS and leads to an elevation of cGMP within the cell. Tamoxifen, an ER antagonist, has been shown to block estrogenstimulated NO release, but not the acute DHEA effect, which was abolished by pertussis toxin. Interestingly, albumin-conjugated DHEA also stimulated NO release, comforting the hypothesis that DHEA exerts its action through a plasma membrane-initiated mechanism, possibly involving a $\mathrm{G}$ protein.

Platelets in patients with type 2 DM are more sensitive to aggregation, which has been attributed to a reduced ability of these cells to produce NO. Recently, DHEA was shown to reduce platelet ADP-induced aggregation by
$40 \%$ in plasma obtained from postmenopausal women with type-2 DM. Using several pharmacologic agents, the authors determined that DHEA action on the platelets was mediated by the activation of the PKC $/ \mathrm{eNOS} / \mathrm{NO} /$ cGMP pathway (44). Similarly, the increased adhesion of U937 monocytes on HUVEC, induced by high glucose concentrations in vitro, as well as the concomitant overexpression of several adhesion molecules was abolished by DHEA (32). Moreover, DHEA completely blocked the oxidative stress and decreased the translocation of NF- $\kappa B$ induced by glucose in these cells, suggesting that DHEA could be useful for preventing some complications of DM and hyperglycemia.

\subsubsection{Mechanisms of Dehydroepiandrosterone (Sulfate) Action on Vascular Smooth Muscle Cells}

Pulmonary arterial hypertension is a severe disease of the pulmonary vasculature, which is defined by an increased arterial pressure and is characterized by proliferation and resistance to apoptosis of arterial smooth muscle cells. This last phenotype is partly due to the activation by growth factors and inflammatory cytokines of neoplastic pathways involving the transcription factor STAT3 under the control of the protein Src. Similar to cancer cells, Src/STAT3 stimulation leads to the activation of NFAT signaling, itself responsible for both proliferation and resistance to apoptosis.

The DHEA (S) has been reported to be a pulmonary vasodilator that prevent acute hypoxic pulmonary vasoconstriction by opening potassium channels (38) and upregulating the NO-modulated soluble guanylyl cyclase (39), both mechanisms leading to relaxation of vascular smooth muscle cells. Recently, DHEA was shown to reduce the activity of voltage-activated T-type calcium channels within various cell types, including pulmonary artery smooth muscle cells. The modifications of the electrophysiologic properties of the calcium channels observed upon DHEA challenge resulted in the inhibition of $\mathrm{KCl}$-induced contraction of pulmonary artery rings. They were independent of AR and ER and a pertussis toxin-sensitive Gi protein pathway is implicated for these effects (40).

In addition, DHEA reverses human vascular remodeling by inhibiting Src/STAT3 constitutive activation (41). This in vitro inhibition by DHEA was shown to be associated with a reduced activity of NFAT, a mitochondrial membrane depolarization (sensitizing the cell to apoptosis), and a decrease of intracellular calcium concentrations, all these factors contributing to a reduction of cell proliferation. The response to DHEA was similar to that observed upon molecular inhibition of STAT3, suggesting STAT3 as a main target for the hormone in smooth muscle cells.

\subsubsection{Specific Effects of Dehydroepiandrosterone on Car- diomyocytes}

The putative cardioprotective action of DHEA, which was suggested by several epidemiologic observational 
studies, was tested in vitro using a neonatal rat cardiomyocyte culture system. It was found that the presence of DHEA significantly inhibited the increase in myocyte cell size and BNP mRNA levels upregulated by endothelin-1 (42). Interestingly, in the same study, the authors reported that DHEA levels in humans, measured at the coronary sinuses and aortic roots during cardiac catheterization, were suppressed in patients with heart failure in contrast to controls. The opposite was observed for aldosterone concentrations, which were increased in heart failure. They postulated that DHEA or its metabolites exert a cardioprotective action through their anti-hypertrophic effects.

Recently, another study investigated the ability of DHEA to counteract the chronotropic and hypertrophic actions of aldosterone and glucocorticoids (43). Freshly isolated neonate rat cardiomyocytes contract spontaneously in culture and their beating frequency is markedly increased upon stimulation of the mineralocorticoid or the glucocorticoid receptors (MR and GR, respectively). DHEA was found to reduce this chronotropic response by decreasing the T-type calcium channel expression and activity within cardiomyocytes. In the same model, DHEA also prevented MR- and GR-mediated myocyte hypertrophy as well as the expression of ANP and BNP. The kinetics of the negative chronotropic action of DHEA and its sensitivity to actinomycin D revealed the presence of both genomic and non-genomic mechanism of action. While the genomic action was mostly related to MR inhibition, the rapid non-genomic response appeared to be due to DHEA antioxidant properties. The reduction of myocyte hypertrophy and spontaneous beating frequency by DHEA is clearly beneficial for the heart function; however, epiandrosterone, a metabolite of DHEA, has been reported to reduce L-type calcium channel activity in ventricular myocytes and therefore, inhibits myocardial contractility (45).

\section{Conclusions}

DHEA (S) is the hormone not only circulating at the highest concentration in blood, but it is also displaying the most extensive decrease upon aging. Therefore, it has been tempting to correlate the reduction of the hormone levels with several types of age-related dysfunctions including CVD. Many observational studies performed over decades, demonstrated the existence of a significant inverse association between levels of DHEA (S) and cardiovascular risk, morbidity, and mortality, even after correcting for usual confounding factors. Nevertheless, a strong correlation is not a proof of causality. In order to discriminate between a hormonal deficiency directly responsible for the observed disease or the hormone acting as a simple marker of the biologic age (possibly the main cause of the increased frequency of pathologies), the problem was investigated by measuring the beneficial effects of hormone supplementation. In other words, the question addressed here was whether DHEA (S) was the anti-aging hormone. Unfortunately, supplementation studies were much less conclusive than expected and the benefit of DHEA (S) on the outcome of CVD is still highly controversial. Discrepancies among results can be due to several factors such as insufficient duration of treatment, heterogeneity of the tested population, irreversibility of investigated pathologies, or even economic concerns. Indeed, because DHEA $(S)$ is a cheap, not patentable molecule, already on the free market for a long time in the United States, there is poor motivation in the industry for conducting rigorous and expensive clinical trials.

Therefore, it is not surprising that the strongest information on DHEA (S) action has been obtained from in vitro studies. However, strong limitation in the discovery of the molecular mechanisms of DHEA (S) action are the lack of a specific receptor, which can be clearly identified as responsible for the hormone response, as well as the ability of DHEA ( $S$ ) to be converted into androgens and estrogens. Moreover, similar to other steroid hormones, DHEA (S) appears to exert its effect through genomic and non-genomic pathways, the latter of which included simple antioxidant properties. Nevertheless, a better comprehension of the basic mechanisms leading to the multiple actions of DHEA (S) on its various target cells will be certainly useful for developing new strategies and better apprehending CVD, which is still killing too many people in Western countries.

\section{Authors' Contributions}

Study concept and design, drafting the manuscript, and critical revision of the manuscript for important intellectual content: Tiphaine Mannic, Joanna Viguie, and Michel Florian Rossier.

\section{Funding/Support}

This study was supported by the Foundation Endocrinology of Geneva (M.F.R and T.M).

\section{References}

1. Pitt B, Remme W, Zannad F, Neaton J, Martinez F, Roniker B, et al. Eplerenone, a selective aldosterone blocker, in patients with left ventricular dysfunction after myocardial infarction. $N$ Engl J Med. 2003;348(14):1309-21.

2. Weiss EP, Villareal DT, Ehsani AA, Fontana L, Holloszy JO. Dehydroepiandrosterone replacement therapy in older adults improves indices of arterial stiffness. Aging Cell. 2012;11(5):876-84

3. Savineau JP, Marthan R, Dumas de la Roque E. Role of DHEA in cardiovascular diseases. Biochem Pharmacol. 2013;85(6):718-26.

4. Mazat L, Lafont S, Berr C, Debuire B, Tessier JF, Dartigues JF, et al Prospective measurements of dehydroepiandrosterone sulfate in a cohort of elderly subjects: relationship to gender, subjective health, smoking habits, and 10-year mortality. Proc Natl Acad Sci U S A. 2001;98(14):8145-50.

5. Kroboth PD, Salek FS, Pittenger AL, Fabian TJ, Frye RF. DHEA and DHEA-S: a review. J Clin Pharmacol.1999;39(4):327-48.

6. Barrett-Connor E, Khaw KT, Yen SS. A prospective study of dehydroepiandrosterone sulfate, mortality, and cardiovascular disease. N Engl J Med.1986;315(24):1519-24.

7. Shufelt C, Bretsky P, Almeida CM, Johnson BD, Shaw LJ, Azziz 
$\mathrm{R}$, et al. DHEA-S levels and cardiovascular disease mortality in postmenopausal women: results from the National Institutes of Health--National Heart, Lung, and Blood Institute (NHLBI)sponsored Women's Ischemia Syndrome Evaluation (WISE). J Clin Endocrinol Metab. 2010;95(11):4985-92.

8. Kedziora-Kornatowska K, Beszczynska-Oles R, Kornatowski T, Szadujkis-Szadurski L. The analysis of dehydroepiandrosterone sulphate concentration in elderly age women depending on coexisting disease states. Adv Med Sci. 2007;52 Suppl 1:12 6-30.

9. Haring R, Teng Z, Xanthakis V, Coviello A, Sullivan L, Bhasin S, et al. Association of sex steroids, gonadotrophins, and their trajectories with clinical cardiovascular disease and all-cause mortality in elderly men from the Framingham Heart Study. Clin Endocrinol (Oxf). 2013;78(4):629-34.

10. Akishita M, Hashimoto M, Ohike Y, Ogawa S, Iijima K, Eto M, et al. Association of plasma dehydroepiandrosterone-sulfate levels with endothelial function in postmenopausal women with coronary risk factors. Hypertens Res. 2008;31(1):69-74.

11. Yoshida S, Aihara K, Azuma H, Uemoto R, Sumitomo-Ueda Y, Yag $\mathrm{S}$, et al. Dehydroepiandrosterone sulfate is inversely associated with sex-dependent diverse carotid atherosclerosis regardless of endothelial function. Atherosclerosis. 2010;212(1):310-5

12. Hak AE, Witteman JC, de Jong FH, Geerlings MI, Hofman A, Pols HA. Low levels of endogenous androgens increase the risk of atherosclerosis in elderly men: the Rotterdam study.J Clin Endocrinol Metab. 2002;87(8):3632-9.

13. Herrington DM. Dehydroepiandrosterone and coronary atherosclerosis. Ann N YAcad Sci. 1995;774:271-80.

14. Feldman HA, Johannes CB, Araujo AB, Mohr BA, Longcope C, McKinlay JB. Low dehydroepiandrosterone and ischemic heart disease in middle-aged men: prospective results from the Massachusetts Male Aging Study. Am J Epidemiol. 2001;153(1):79-89.

15. Jimenez MC, Sun Q, Schurks M, Chiuve S, Hu FB, Manson JE, et al. Low dehydroepiandrosterone sulfate is associated with increased risk of ischemic stroke among women. Stroke. 2013;44(7):1784-9.

16. Ravaglia G, Forti P, Maioli F, Sacchetti L, Nativio V, Scali CR, et al. Dehydroepiandrosterone-sulfate serum levels and common agerelated diseases: results from a cross-sectional Italian study of a general elderly population. Exp Gerontol. 2002;37(5):701-12.

17. Liu L, Wang M, Yang X, Bi M, Na L, Niu Y, et al. Fasting serum lipid and dehydroepiandrosterone sulfate as important metabolites for detecting isolated postchallenge diabetes: serum metabolomics via ultra-high-performance LC-MS. Clin Chem. 2013;59(9):1338-48.

18. Suzuki T, Yano Y, Sakamoto M, Uemura M, Yasuma T, Onishi Y, et al. Correlation of circulating dehydroepiandrosterone with activated protein $C$ generation and carotid intima-media thickness in male patients with type 2 diabetes. Diabet Med.2012;29(7):e416

19. Ponikowska B, Jankowska EA, Maj J, Wegrzynowska-Teodorczyk K, Biel B, Reczuch K, et al. Gonadal and adrenal androgen deficiencies as independent predictors of increased cardiovascular mortality in men with type II diabetes mellitus and stable coronary artery disease. Int J Cardiol. 2010;143(3):343-8.

20. Bonnet S, Paulin R, Sutendra G, Dromparis P, Roy M, Watson KO, et al. Dehydroepiandrosterone reverses systemic vascular remodeling through the inhibition of the Akt/GSK3-\{beta\}/NFAT axis. Circulation. 2009;120(13):1231-40.

21. Aragno M, Meineri G, Vercellinatto I, Bardini P, Raimondo S, Peiretti PG, et al. Cardiac impairment in rabbits fed a high-fat diet is counteracted by dehydroepiandrosterone supplementation. Life Sci. 2009;85(1-2):77-84.

22. Aragno M, Mastrocola R, Alloatti G, Vercellinatto I, Bardini P, Geuna $\mathrm{S}$, et al. Oxidative stress triggers cardiac fibrosis in the heart of diabetic rats. Endocrinology. 2008;149(1):380-8.

23. Aragno M, Mastrocola R, Medana C, Catalano MG, Vercellinatto I, Danni O, et al. Oxidative stress-dependent impairment of cardiac-specific transcription factors in experimental diabetes. Endocrinology. 2006;147(12):5967-74

24. Dumas de La Roque E, Bellance N, Rossignol R, Begueret H, Billaud M, dos Santos P, et al. Dehydroepiandrosterone reverses chronic hypoxia/reoxygenation-induced right ventricular dys- function in rats. Eur Respir J. 2012;40(6):1420-9.

25. Kawano H, Yasue H, Kitagawa A, Hirai N, Yoshida T, Soejima H, et al. Dehydroepiandrosterone supplementation improves endothelial function and insulin sensitivity in men.J Clin Endocrino Metab. 2003;88(7):3190-5.

26. Williams MR, Dawood T, Ling S, Dai A, Lew R, Myles K, et al. Dehydroepiandrosterone increases endothelial cell proliferation in vitro and improves endothelial function in vivo by mechanisms independent of androgen and estrogen receptors. J Clin Endocrinol Metab. 2004;89(9):4708-15.

27. McHenry CM, Bell PM, Hunter SJ, Thompson CJ, Courtney $\mathrm{CH}$ Ennis $\mathrm{CN}$, et al. Effects of dehydroepiandrosterone sulphate (DHEAS) replacement on insulin action and quality of life in hypopituitary females: a double-blind, placebo-controlled study Clin Endocrinol (Oxf). 2012;77(3):423-9.

28. Goel RM, Cappola AR. Dehydroepiandrosterone sulfate and postmenopausal women. Curr Opin Endocrinol Diabetes Obes. 2011;18(3):171-6

29. Sahelian R, Borken S. Dehydroepiandrosterone and cardiac arrhythmia. Ann Intern Med.1998;129(7):588.

30. Curatola AM, Huang K, Naftolin F. Dehydroepiandrosterone (DHEA) inhibition of monocyte binding by vascular endothelium is associated with sialylation of neural cell adhesion molecule. Reprod Sci. 2012;19(1):86-91.

31. Gutierrez G, Mendoza C, Zapata E, Montiel A, Reyes E, Montano LF, et al. Dehydroepiandrosterone inhibits the TNF-alpha-induced inflammatory response in human umbilical vein endothelia cells. Atherosclerosis. 2007;190(1):90-9.

32. Huerta-Garcia E, Ventura-Gallegos JL, Victoriano ME, MontielDavalos A, Tinoco-Jaramillo G, Lopez-Marure R. Dehydroepiandrosterone inhibits the activation and dysfunction of endothelial cells induced by high glucose concentration. Steroids 2012;77(3):233-40.

33. Altman R, Motton DD, Kota RS, Rutledge JC. Inhibition of vascular inflammation by dehydroepiandrosterone sulfate in human aortic endothelial cells: roles of PPARalpha and NF-kappaB. Vascul Pharmacol. 2008;48(2-3):76-84

34. Zapata E, Ventura JL, De la Cruz K, Rodriguez E, Damian P, Masso $\mathrm{F}$, et al. Dehydroepiandrosterone inhibits the proliferation of human umbilical vein endothelial cells by enhancing the expression of p53 and p21, restricting the phosphorylation of retinoblastoma protein, and is androgen- and estrogen-receptor independent. FEBS J. 2005;272(6):1343-53.

35. Nheu L, Nazareth L, Xu GY, Xiao FY, Luo RZ, Komesaroff P, et al. Physiological effects of androgens on human vascular endothelial and smooth muscle cells in culture. Steroids. 2011:76(14):1590-6.

36. Liu D, Dillon JS. Dehydroepiandrosterone stimulates nitric oxide release in vascular endothelial cells: evidence for a cell surface receptor. Steroids. 2004;69(4):279-89.

37. Bhuiyan MS, Tagashira H, Fukunaga K. Dehydroepiandrosteronemediated stimulation of sigma-1 receptor activates Akt-eNOS signaling in the thoracic aorta of ovariectomized rats with abdominal aortic banding. Cardiovasc Ther. 2011;29(4):219-30.

38. Gupte SA, Li KX, Okada T, Sato K, Oka M. Inhibitors of pentose phosphate pathway cause vasodilation: involvement of voltagegated potassium channels. J Pharmacol Exp Ther. 2002;301(1):299305

39. Oka M, Karoor V, Homma N, Nagaoka T, Sakao E, Golembeski SM, et al. Dehydroepiandrosterone upregulates soluble guanylate cyclase and inhibits hypoxic pulmonary hypertension. Cardiovasc Res. 2007;74(3):377-87.

40. Chevalier M, Gilbert G, Lory P, Marthan R, Quignard JF, Savineau IP. Dehydroepiandrosterone (DHEA) inhibits voltage-gated Ttype calcium channels. Biochem Pharmacol. 2012;83(11):1530-9.

41. Paulin R, Meloche J, Jacob MH, Bisserier M, Courboulin A, Bonnet S. Dehydroepiandrosterone inhibits the Src/STAT3 constitutive activation in pulmonary arterial hypertension. Am J Physiol Heart Circ Physiol. 2011;301(5):H1798-809.

42. Nakamura S, Yoshimura M, Nakayama M, Ito T, Mizuno Y, Harada $\mathrm{E}$, et al. Possible association of heart failure status with synthetic balance between aldosterone and dehydroepiandrosterone in human heart. Circulation. 2004;110(13):1787-93.

43. Mannic T, Mouffok M, Python M, Yoshida T, Maturana AD, Vuil- 


\section{Mannic Tet al.}

leumier N, et al. DHEA prevents mineralo- and glucocorticoid receptor-induced chronotropic and hypertrophic actions in isolated rat cardiomyocytes. Endocrinology. 2013;154(3):1271-81.

44. Munoz YC, Gomez GI, Moreno M, Solis CL, Valladares LE, Velarde V. Dehydroepiandrosterone prevents the aggregation of platelets obtained from postmenopausal women with type 2 diabetes mellitus through the activation of the PKC/eNOS/NO pathway. Horm Metab Res. 2012;44(8):625-31.

45. Gupte SA, Tateyama M, Okada T, Oka M, Ochi R. Epiandrosterone, a metabolite of testosterone precursor, blocks L-type calcium channels of ventricular myocytes and inhibits myocardial contractility.J Mol Cell Cardiol.2002;34(6):679-88. 